\title{
PREDIÇÃO DA ADESÃO AO TRATAMENTO E QUALIDADE DE VIDA DE PACIENTES COM TRANSTORNO BIPOLAR
}

\section{PREDICTION OF TREATMENT ADHERENCE AND QUALITY OF LIFE OF PATIENTS WITH BIPOLAR DISORDER}

\section{PREDICCIÓN DE LA ADHERENCIA AL TRATAMIENTO Y CALIDAD DE VIDA DE PACIENTES CON TRASTORNO BIPOLAR}

Clarice de Lourdes Enes ${ }^{1}$, Pedro Henrique Batista de Freitas² ${ }^{2}$ Sebastião Júnior Henrique Duarte ${ }^{3}$, Marco Túlio Resende Clementino ${ }^{4}$, Aline Esteves Pacheco5, Richardson Miranda Machado 6

\section{RESUMO}

Objetivo: Descrever a predição de adesão ao tratamento e a percepção de qualidade de vida de pacientes com transtorno bipolar. Métodos: Estudo transversal realizado com 35 pacientes de um Centro de Atenção Psicossocial III. Foi utilizada uma escala de avaliação da predição da adesão ao tratamento e outra, para avaliar a qualidade de vida dos pacientes, realizou-se análise descritiva dos dados. Resultados: Observou-se maior predição de adesão nos aspectos relacionados à aliança terapêutica com os profissionais $(91,4 \%)$; dificuldade em seguir o tratamento e efeitos adversos da medicação impactaram negativamente $(91,4 \%)$. A maioria (77,2\%) apresentou média de qualidade de vida regular. A qualidade de vida foi melhor no domínio relações sociais (31,5\%) e pior no domínio meio ambiente $(25,7 \%)$. Conclusão: É necessário construir estratégias de cuidado, pela Enfermagem, que minimizem os desconfortos da medicação e que promovam a reabilitação psicossocial, com o intuito de potencializar a adesão e promover a qualidade de vida dos pacientes.

Descritores: Enfermagem; Transtorno bipolar; Serviços de saúde mental; Cooperação e adesão ao Tratamento; Qualidade de vida.

\section{ABSTRACT}

Objective: To describe the prediction of treatment adherence and quality of life of patients with bipolar disorder. Methods: crosssectional study with 35 patients from a Psychosocial Care Center III. Scales were used to assess the prediction of treatment adherence and the quality of life of the patients. Results: there was a higher prediction of adherence in aspects related to therapeutic alliance with professionals $(91.4 \%)$, while difficulty in following treatment and adverse effects of medication caused a negatively impact (91.4\%). Most (77.2\%) had an average of regular quality of life. Quality of life was better in the social relations domain (31.5\%) and worse in the environment domain (25.7\%). Conclusion: it is necessary to build nursing care strategies that minimize medication discomfort and promote psychosocial rehabilitation, in order to enhance adherence and promote patients' quality of life. Descriptors: Nursing; Bipolar Disorder; Mental Health Services; Cooperation and Adherence to Treatment; Quality of Life.

\section{RESUMEN}

Objetivo: describir la predeción de la adherencia al tratamiento y la calidad de vida de los pacientes con trastorno bipolar. Métodos: estudio transversal realizado con 35 pacientes de un Centro de Atención Psicosocial III. Se utilizaron escalas para la evaluación de la predicción de la adherencia al tratamiento y otra para evaluar la calidad de vida de los pacientes, se realizó el análisis descriptivo de los datos. Resultados: se observó mayor predicción de adherencia en los aspectos relacionados a la alianza terapéutica con los profesionales (91,4\%); dificultad para seguir el tratamiento y efectos adversos de la medicación impactaron negativamente (91,4\%). La mayoría (77,2\%) presentó promedio de calidad de vida regular. La calidad de vida fue mejor en el dominio de relaciones sociales $(31,5 \%)$, y peor en el dominio de medio ambiente $(25,7 \%)$. Conclusión: es necesario desarrollar estrategias de atención de enfermería que minimicen la incomodidad de los medicamentos y promuevan la rehabilitación psicosocial, para mejorar la adherencia y promover la calidad de vida de los pacientes.

Descriptores: Enfermería; Trastorno Bipolar; Servicios de Salud Mental; Cooperación y Adherencia al Tratamiento; Calidad de Vida.

${ }^{1}$ Psicóloga, Mestre em Ciências da Saúde pelo Programa de Pós-Graduação em Enfermagem da Universidade Federal de São João Del-Rei. ${ }^{2}$ Enfermeiro, Mestre e Doutorando Programa de Pós-Graduação em Ciências da Saúde da Universidade Federal de São João Del-Rei. ${ }^{3}$ Enfermeiro, Mestre e Doutor em Ciências da Saúde. Professor Associado da Universidade Federal de Mato Grosso do Sul. ${ }^{4}$ Assistente Social, Mestrando do Programa de Pós-Graduação em Enfermagem da Universidade Federal de São João Del-Rei. ${ }^{5}$ Enfermeira, Mestre em Ciências da Saúde pelo Programa de Pós-Graduação em Enfermagem da Universidade Federal de São João Del-Rei. ${ }^{6}$ Enfermeiro, Mestre e Doutor em Psiquiatria. Professor Associado da Universidade Federal de São João Del-Rei.

\section{Como citar este artigo:}

Enes CL, Freitas PHB, Duarte SJH, et al.Predição da adesão ao tratamento e qualidade de vida de pacientes com transtorno bipolar. Revista de Enfermagem do Centro oeste Mineiro 2020;10:e3489.[Access ]. Available in: DOI: 


\section{INTRODUÇÃO}

O transtorno bipolar é uma doença mental crônica e recorrente, caracterizada por quadros episódicos de oscilações de humor que se manifestam sob as formas de depressão e mania (euforia - tipo I) ou hipomania (tipo II), com diferentes graus de intensidade, sucedidos ou intercalados por períodos de remissão. Possui etiologia complexa e multifatorial, sendo resultado da interação de fatores genéticos, psicossociais e ambientais. É responsável por impactos econômicos e sociais, contribuindo para o aumento da utilização dos serviços e dos gastos em saúde ${ }^{(1)}$.

Em função de suas repercussões, esse transtorno é considerado um dos distúrbios psiquiátricos mais graves e prevalentes. Dados globais indicaram que, entre 1990 e 2013, houve um aumento de 32,7 milhões para 48,8 milhões $(49,1 \%)$ no número de casos prevalentes. A idade média dos primeiros sintomas é de 25 anos, embora possa surgir mais tarde, após os 40 anos ${ }^{(2)}$. Ocorre sem distinção de raça, etnia e classe social, porém com séria distinção na magnitude entre os sexos. No Brasil, o transtorno bipolar apresenta perfil epidemiológico similar, com prevalência em torno de 1 a $4 \%$ da população, início dos sintomas antes dos 30 anos, sendo mais prevalente em mulheres ${ }^{(3)}$. Considera-se que o transtorno bipolar possa aumentar em até 20 vezes o risco de suicídio e, em função da idade de início dos sintomas, também pode reduzir a expectativa de vida entre 11 e 20 anos $^{(2)}$.

As principais terapêuticas direcionadas ao transtorno bipolar centram-se em intervenções farmacológicas e psicossociais para o controle dos episódios agudos e manutenção do quadro, em longo prazo. $O$ tratamento farmacológico contínuo configura-se como uma condição essencial para melhor prognóstico, resultando na diminuição dos sintomas maníacos, frequência dos episódios agudos e da alternância do humor ${ }^{(4)}$. Nesse sentido, a política atual de saúde mental preconiza que os casos graves e persistentes de transtornos mentais sejam tratados nos Centros de Atenção Psicossocial (CAPS), serviços abertos e de base comunitária que propõem, paralelamente, estratégias comunitárias para a inclusão social e, no Centro de Atenção Psicossocial tipo III (CAPS III), leitos de acolhimento e observação noturna para casos de crise psíquica ${ }^{(5)}$.

Dentro desse contexto, emerge a necessidade de adesão ao tratamento como um dos principais pilares para a melhora dos sintomas e da qualidade de vida do paciente. A eficácia do tratamento está relacionada à adesão, compreendida como o cumprimento das recomendações clínicas pelo paciente. No entanto, estudos sobre adesão em doenças crônicas, incluindo transtornos psiquiátricos, demonstram que os pacientes apresentam dificuldades em seguir as prescrições por fatores clínicos, econômicos, culturais e sociais, o que leva à piora da doença ${ }^{(6)}$. Ademais, estudos sobre adesão ao tratamento farmacológico e seus resultados clínicos devem ser analisados com cautela, tendo em vista as limitações metodológicas e altas taxas de abandono, dificultando a generalização dos resultados ${ }^{(7)}$.

No Brasil, a adesão ao tratamento de pacientes com transtorno bipolar tem sido pesquisada, especialmente, em condições nas quais os mesmos já se encontram inseridos em seu contexto social e familiar, após a alta do tratamento da crise aguda. Em geral, são avaliados em serviços ambulatoriais de saúde mental destinados ao acompanhamento e manutenção do tratamento ${ }^{(8)}$. Todavia, há escassez de estudos que estimem a predição de adesão ao tratamento do transtorno bipolar, no momento da alta do paciente, verificada por meio da revisão de literatura, emergindo uma importante lacuna. 0 conhecimento dos fatores preditivos de adesão ou não adesão podem se configurar como ferramentas indispensáveis para estruturar e reorganizar as ações dos serviços de saúde em todos os seus níveis, de forma a contribuir para a estabilidade do paciente e, consequentemente, para redução das recaídas e reinternações ${ }^{(9)}$.

Diante do exposto, o presente estudo objetivou descrever a predição de adesão ao tratamento e a percepção de qualidade de vida de pacientes com transtorno bipolar.

\section{MÉTODOS}

Trata-se de um estudo transversal realizado no CAPS III, de um município de médio porte, localizado na região Centro-Oeste do Estado de Minas Gerais. Segundo o IBGE, a população estimada para o município em 2019 é de 238.230 habitantes, e o referido serviço presta assistência a uma população de cerca de 300.000 habitantes, incluindo cinco municípios vizinhos, configurandose em referência, na região, por meio de pactuação intermunicipal. De acordo com o DATASUS, a rede de atenção psicossocial do município é constituída, além do referido CAPS III, 
por um CAPS Álcool e Drogas III (CAPS AD III), um Serviço Residencial Terapêutico (SRT), uma Unidade de Pronto Atendimento (UPA) que presta assistência de retaguarda e 36 unidades de saúde da família.

A população do estudo foi composta pelos pacientes internados com diagnóstico de transtorno afetivo bipolar, entre fevereiro de 2017 e fevereiro de 2018, no CAPS III para tratamento intensivo, em decorrência da crise. Para a constituição da amostra, optou-se por empregar uma amostragem não probabilística por conveniência do subtipo acidental, ou seja, não foi estabelecido cálculo amostral estatístico, pois para avaliar a predição da adesão ao tratamento proposto seria viável realizar com pacientes que foram internados e no momento da alta ${ }^{(10)}$. Desse modo, sendo um número não previsível e somente conhecido, a partir do acompanhamento da ocorrência em determinado período.

Foram adotados, como critérios de inclusão, o paciente ter diagnóstico de transtorno afetivo bipolar estabelecido pelo médico psiquiatra assistente; idade igual ou superior a 18 anos; ser capaz de se comunicar verbalmente; apresentar quadro estabilizado e estar com alta do tratamento intensivo definida pela equipe de referência. Adotou-se, como critério de exclusão, o paciente ter interrompido ou abandonado o tratamento.

A coleta de dados ocorreu, durante todo o período do estudo, a partir da comunicação da alta do paciente aos pesquisadores, pelo técnico de referência do tratamento do paciente no CAPS III, sendo o momento específico o dia da alta psiquiátrica da modalidade de tratamento intensivo. No período estabelecido, 168 pacientes deram entrada no serviço para internação, sendo 41 com diagnóstico de transtorno bipolar. A amostra final constituiu-se de 35 pacientes que atenderam aos critérios de inclusão.

Para a coleta dos dados, foram utilizadas como instrumentos duas escalas, sendo uma para prever a adesão ao tratamento proposto para o transtorno bipolar e outra, para avaliar a percepção do paciente quanto à sua qualidade de vida. Ambas as escalas são validadas e de acesso livre e gratuito.

A Escala Clínica para Prever a Adesão ao Tratamento no Transtorno Bipolar do Humor (ECPAT-TBH) é um instrumento validado no Brasil $^{(11)}$, constituído por 21 itens que correspondem aos fatores mais relevantes no processo de adesão e não adesão ao tratamento pelos pacientes. Permite avaliar a predição da adesão à terapêutica farmacológica e psicossocial proposta, possibilitando identificar fatores impactantes no comportamento do paciente bipolar em aderir ao tratamento. Além disso, apresenta coeficientes de validade e precisão satisfatórios e um coeficiente alpha de Cronbach de 0,79 .

Já a Escala de Avaliação da Qualidade de Vida (World Health Organization Quality of Life Assessment-bref, ou WHOQOL-brefl é um instrumento composto por 26 questões fechadas, sendo duas de caráter geral, que avaliam a percepção da qualidade de vida e a satisfação com a saúde pelo paciente. As outras 24 questões são distribuídas em quatro domínios (Físico, Psicológico, Relações Sociais e Meio Ambiente), representados, cada um, por várias facetas. A pontuação das questões é obtida por meio de uma escala do tipo Likert de cinco pontos (1 a 5). Optou-se, neste estudo, por agrupar os pacientes em três classes de distribuição de frequência: Ruim (de 1,0 a 2,9); Regular (de 3,0 a 3,9); e Boa (de 4,0 a 4,9). Ao final da aplicação da escala, cada domínio teve seu escore calculado, individualmente, o que permitiu a avaliação do nível de qualidade de vida em cada contexto. Desse modo, quanto mais alto o escore melhor a qualidade de vida em cada domínio.

Ainda, por meio desses instrumentos, foi realizada a coleta de dados no dia da alta do paciente. Ao preencher os critérios de inclusão, o paciente foi, assim, convidado a participar da pesquisa e, ao aceitar, reuniu-se, individualmente, com um dos pesquisadores, sendo-lhe apresentada a proposta do estudo e as questões abordadas nos instrumentos a serem respondidas. Após o esclarecimento de possíveis dúvidas, o paciente assinou o Termo de Consentimento Livre e Esclarecido (TCLE). Em seguida, foram aplicadas as escalas para avaliação da predição da adesão ao tratamento e da qualidade de vida. As perguntas dos instrumentos foram lidas para o paciente e as respostas registradas pelo pesquisador. Foi esclarecido aos entrevistados que o sigilo e o anonimato das informações seriam preservados.

Todos os dados obtidos nas coletas foram tabulados e tratados no programa Excel e, após a organização dos mesmos, foi realizada a análise de consistência. Adicionalmente, utilizou-se o software Statistical Package for the Social Sciences, versão 21 . Foram conduzidas análises descritivas, por meio de medidas de frequências (absoluta e relativa) e tendência central (média). 
Os dados coletados obedeceram toda a proteção e segurança estabelecidas pelo Comitê de Ética em Pesquisa (CEP) da Universidade Federal de São João Del-Rei, Campus Centro-Oeste Dona Lindu, em Divinópolis, Minas Gerais. O processo seguiu os termos da Resolução no 466/2012 do Conselho Nacional de Saúde, que trata de normas sobre a pesquisa envolvendo seres humanos. $O$ estudo foi aprovado no CEP sob o parecer de no 1.868 .647 (CAAE: 60788516.0000.5545).

\section{RESULTADOS E DISCUSSÃO}

A caracterização sociodemográfica e clínica mostrou predominância de pacientes do sexo feminino $(68,6 \%)$, idade entre 41 e 50 anos $(31,4 \%)$, solteiros $(62,9 \%)$, ensino fundamental (57,1\%) e aposentados (57,1\%), em tratamento há mais de 10 anos $(74,3 \%)$ e uso de mais de 4 tipos de medicamentos/dia (82,9\%).Observou-se, durante a coleta dos dados, a reinternação de 12 dos 35 pacientes avaliados no estudo. Dessa forma, foi realizada nova coleta de dados com esses pacientes e foi considerada válida apenas esta última.

A Tabela 1 apresenta a predição de adesão/não adesão dos pacientes ao tratamento proposto na alta da internação, considerando as médias de escores obtidas para os diferentes grupos de questões da escala de predição de adesão. Os resultados obtidos indicaram uma tendência geral dos pacientes em aderir ao tratamento proposto, no momento da alta $(n=27$, ou $77,1 \%$ ). Verificou-se que a predição de adesão foi maior no Grupo 5 (91,4\%), Tratamento Psicossocial e Aliança Terapêutica com os Profissionais e Serviços de Saúde Mental, e no Grupo 3 (88,6\%), Aceitação do Transtorno Bipolar pelo Paciente, Familiar ou Cuidador e pela Comunidade. Em contraposição, o Grupo 6 (91,4\%), Dificuldade em Seguir o Tratamento e Efeitos Adversos da Medicação, foi impactado mais negativamente na predição de adesão.

Tabela 1 - Distribuição da frequência dos pacientes com transtorno bipolar $(n=35)$ quanto à predição da adesão ao tratamento proposto na alta da internação. Minas Gerais, 2018.

\begin{tabular}{lcc}
\multicolumn{1}{c}{ Grupos } & $\begin{array}{c}\text { Adesão } \\
\mathbf{n}(\%)\end{array}$ & $\begin{array}{c}\text { Não adesão } \\
\mathbf{n}(\%)\end{array}$ \\
\hline \hline Grupo 1 (Comportamento e Sentimento Diante do Tratamento Medicamentoso) & $20(57,1)$ & $15(42,9)$ \\
Grupo 2 (Contribuição da Religião no Enfrentamento do Transtorno Bipolar) & $18(51,4)$ & $17(48,6)$ \\
Grupo 3 (Aceitação do Transtorno Bipolar pelo Paciente, Familiar ou Cuidador, e pela & $31(88,6)$ & $4(11,4)$ \\
Comunidade) & $21(60,0)$ & $14(40,0)$ \\
Grupo 4 (Manutenção de Vida Social e Comorbidades pelo Uso de Substâncias) & $32(91,4)$ & $3(8,6)$ \\
$\begin{array}{l}\text { Grupo } 5 \text { (Adesão ao Tratamento Psicossocial e Aliança Terapêutica com os Profissionais e Serviços } \\
\text { de Saúde Mental) }\end{array}$ & $3(8,6)$ & $32(91,4)$ \\
Grupo 6 (Dificuldade em Seguir o Tratamento e Efeitos Adversos da Medicação) & $27(77,1)$ & $8(22,9)$ \\
Predição Geral & &
\end{tabular}

Fonte: Dados da pesquisa, 2018.

A qualidade de vida dos pacientes foi avaliada, tanto em seu aspecto geral quanto por domínios, obtendo-se níveis predominantemente regulares para ambos os aspectos. A distribuição de frequência dos pacientes, de acordo com níveis gerais de classificação de qualidade de vida, realizada seguindo-se os indicadores da escala WHOQOL-bref, mostrou que a maioria dos 35 pacientes estudados $(77,2 \%)$ apresentou qualidade de vida regular, sendo os valores médios de escore entre 3,0 e 3,9 (Tabela 2).

Tabela 2 - Distribuição de frequência dos pacientes segundo a classificação de qualidade de vida da escala WHOQOL-bref. Minas Gerais, 2018.

\begin{tabular}{cc}
\hline \hline $\begin{array}{c}\text { Qualidade de vida } \\
\text { (escore médio) }\end{array}$ & $\begin{array}{c}\text { Frequência } \\
\mathbf{n}(\%)\end{array}$ \\
\hline Ruim (até 2,9) & $6(17,1)$ \\
Regular (3,0 a 3,9) & $27(77,2)$ \\
Boa ( $\geq 4,0)$ & $2(5,7)$ \\
Total & $35(100,0)$ \\
\hline \hline
\end{tabular}

Fonte: Dados da pesquisa, 2018. 
A Tabela 3 apresenta a distribuição da frequência por domínios dos pacientes quanto à qualidade de vida. Os domínios Psicológico $(68,6 \%)$ e Físico $(65,7 \%)$ foram os que mais contribuíram para a qualidade de vida regular, seguidos do domínio Meio Ambiente com pior índice para a qualidade de vida dos pacientes $(25,7 \%)$. Por outro lado, o domínio Relações Sociais $(37,1 \%)$ foi considerado o que mais contribuiu para uma boa qualidade de vida.

Tabela 3 - Distribuição de frequência dos pacientes, segundo domínios de qualidade de vida da escala WHOQOL-bref. Minas Gerais, 2018.

\begin{tabular}{lccc}
\hline \multicolumn{1}{c}{ Qualidade de Vida (Domínios) } & Ruim & Regular & Boa \\
\cline { 2 - 4 } & $\mathbf{n}(\%)$ & $\mathbf{n}(\%)$ & $\mathbf{n ( \% )}$ \\
\hline Geral (auto percepção e satisfação com a saúde) & $5(14,3)$ & $17(48,6)$ & $11(31,5)$ \\
Domínio Físico & $7(20,0)$ & $23(65,7)$ & $5(14,3)$ \\
Domínio Psicológico & $6(17,1)$ & $24(68,6)$ & $5(14,3)$ \\
Domínio Relações Sociais & $6(17,1)$ & $18(51,4)$ & $13(37,1)$ \\
Domínio Meio Ambiente & $9(25,7)$ & $24(68,6)$ & $2(5,7)$ \\
\hline \hline
\end{tabular}

Fonte: Dados da pesquisa, 2018.

No que se refere à caraterização dos sujeitos do estudo, identificou-se que a maioria era do sexo feminino $(68,6 \%)$ e solteiro $(62,9 \%)$. Globalmente, evidências apontam magnitudes diferentes entre taxas de prevalência do transtorno bipolar em relação aos tipos I (mais prevalente em homens) e II (mais prevalente em mulheres), embora, de modo geral, a prevalência do transtorno bipolar seja semelhante para ambos os sexos ${ }^{(2,12)}$. A presença de maior número de mulheres, no estudo, pode ser explicada pelas diferenças relativas aos aspectos sociais, psicológicos, fisiológicos e de manifestação do curso da doença que se diferem entre os gêneros e levam as mulheres a uma maior procura por tratamento. Quanto à situação conjugal dos entrevistados, foi observado percentual mais elevado de indivíduos sem parceiro, corroborando dados da literatura que descrevem taxas maiores de solteiros entre portadores de transtorno bipolar, quando comparados à população geral, possivelmente decorrentes das limitações desencadeadas pela doença, como impotência sexual (pelo uso de fármacos), oscilações do humor e dificuldades para cuidar dos filhos ${ }^{(1)}$.

A tendência geral $(n=27$, ou $77,1 \%$ ) dos pacientes em aderir ao tratamento proposto pode ser atribuída ao caráter preditivo do instrumento, que é aplicado no momento da alta da internação quando o paciente se encontra assintomático, com seu quadro clínico estabilizado, estando mais disposto a manter os efeitos benéficos do tratamento. Outra questão relevante relaciona-se, possivelmente, ao instrumento ter abordado a tendência à adesão de forma ampla, não restrita apenas à ótica da medicação, mas considerando também aspectos psicossociais, relacionais, religiosos, igualmente determinantes para a adesão.

A maior predição de adesão $(91,4 \%)$ referente ao tratamento psicossocial e aliança terapêutica com os profissionais e serviços de saúde mental e aceitação do transtorno bipolar pelo paciente, familiar ou cuidador e pela comunidade $(88,6 \%)$, pode estar relacionada ao fato de que a aceitação da doença é construída ao longo do processo do adoecimento e do tratamento. Tendo em vista a necessidade de informações e conhecimento sobre essas questões que são trabalhados pela equipe de saúde mental ${ }^{(13)}$.

O conceito do tratamento-aliança como um vínculo colaborativo e afetivo entre profissionais e pacientes é considerado na literatura psicoterapêutica ${ }^{(6,9)}$ como um dos principais fatores que contribuem para a adesão ao tratamento ${ }^{(6)}$, sendo, assim, compreendido como o envolvimento ativo dos pacientes na tomada de decisão e abordagens baseadas na sua participação e concordância para melhor adesão ao tratamento ${ }^{(12)}$. Um dos aspectos fundamentais dessa estratégia de cuidado consiste no paciente sentir-se acolhido e reconhecer a existência de uma escuta por parte da equipe, principalmente pelos profissionais da enfermagem, os quais passam a maior parte do tempo com os pacientes $^{(15)}$.

A família também possui papel importante no processo de aceitação do tratamento pelo paciente. Estudos ${ }^{(10,15)}$ demonstram que atitudes e 
crenças de saúde de membros da família, seu conhecimento sobre a doença e o tratamento possuem influência direta no modo como o paciente percebe e lida com o seu problema de saúde mental ${ }^{(14)}$. Esse achado pode refletir uma percepção do paciente estabilizado após a melhora da crise, quanto à sua capacidade de melhor aceitação pessoal e social. Por outro lado, estudos $^{(16,19)}$ relatam dificuldades de pacientes e familiares em aceitar o diagnóstico inicial do transtorno bipolar, em razão de estigmas relacionados à doença, o que prejudica 0 tratamento e a manutenção do quadro clínico.

Os efeitos adversos da medicação foram apontados como o maior fator de risco para a não adesão ao tratamento pelos pacientes $(91,4 \%)$, corroborando resultados de outros estudos que apontaram não adesão ao tratamento de $60 \%$ a $90 \%$, devido as reações das medicações ${ }^{(13,17)}$.

A administração de medicamento é uma das maiores responsabilidades da Enfermagem e demais integrantes da equipe de saúde envolvidos no cuidado do paciente. Desse modo, é preciso compreender que não é somente uma tarefa mecânica a ser executada em complacência rígida com a prescrição. Requer pensamento crítico e o exercício de juízo profissional, pois o grande número de efeitos colaterais e reações adversas das medicações psiquiátricas, tanto aquelas administradas por via oral quanto intramuscular, destacam que o enfermeiro possui um importante papel na decisão de administração da medicação. Contudo, nem sempre isso é feito por esse profissional que, por vezes, delega essa decisão à equipe de técnicos e auxiliares de enfermagem ${ }^{(17,19)}$.

Por conta de tais efeitos e reações, ao optar por essa conduta, a equipe de enfermagem deve estar atenta aos sinais e sintomas que os pacientes apresentam após a mesma, implicando tanto na constante avaliação de sinais vitais, independente de diagnóstico prévio de alguma comorbidade, quanto na observação do comportamento que o paciente virá a manifestar. Os medicamentos são, sem dúvida, um forte aliado no tratamento dos pacientes da clínica psiquiátrica. Contudo, devem servir como mediadores para uma melhor interação entre profissionais e pacientes, facilitando o relacionamento terapêutico e a adoção de cuidados que busquem a reabilitação e a valorização da subjetividade de cada indivíduo em tratamento, proporcionando uma assistência de enfermagem voltada para a participação dos pacientes no tratamento e sua autonomia ${ }^{(10)}$.
Desse modo, a escuta qualificada deve ser importante constitutivo na prática da Enfermagem Psiquiátrica. A equipe de enfermagem deve valorizar a narrativa do paciente, suas queixas, sugestões e necessidades. A escuta deve sair do lugar de suspeita da fala e ir para o lugar de testemunha. Antes de pensar em qualquer tipo de cuidado ou intervenção, o enfermeiro deve ouvir e compartilhar com o sujeito em sofrimento. $O$ estabelecimento de um relacionamento terapêutico entre enfermeiro e paciente, permite assim uma assistência de enfermagem pautada no sujeito, trabalhando sua subjetividade de maneira empática, em períodos crônicos ou agudos. Sendo assim, sintomas como agitação, insônia e irritabilidade podem ser mais bem compreendidos e utilizados como guia para o cuidado de enfermagem ${ }^{(10,15)}$.

É importante destacar que cuidar de uma pessoa com doença mental é uma tarefa que exige pensar a pessoa com vontades próprias, com possibilidades de dizer sim, de dizer não e dizer nada para a intervenção ou o cuidado de enfermagem. É preciso que a enfermagem, ao planejar sua assistência, considere que a pessoa, objeto do cuidado, participe da elaboração, manutenção e avaliação do seu tratamento ${ }^{(13,19)}$.

Sendo assim, a dificuldade de aderir ao tratamento em decorrência dos efeitos adversos da medicação serve como um alerta, principalmente para a equipe de enfermagem, que aponta para a necessidade de uma maior participação desses profissionais nos processos decisórios que envolvem a administração medicamentosa. Os efeitos colaterais e adversos das medicações neurolépticas são frequentes, o que torna essencial à prática da Enfermagem Psiquiátrica o estabelecimento de critérios clínicos que indiquem a necessidade ou não de administrar as medicações prescritas ${ }^{(9,12)}$.

É importante ressaltar que a eficácia dos psicofármacos na melhora dos episódios de depressão e mania, bem como na manutenção do tratamento em longo prazo, é consenso na literatura. Por outro lado, seus efeitos adversos associam-se a alterações na libido, sedação e prostração, afetando os pacientes em suas atividades cotidianas e acarretando-lhes prejuízos funcionais $^{(8)}$. Embora essa relação seja reconhecida, estudos ${ }^{(16,19)}$ indicam que essa problemática é mais complexa e pode refletir a oposição entre o impacto dos efeitos adversos e a aceitação dos desconfortos desses efeitos em troca do benefício medicamentoso. Dessa forma, 
o equilíbrio entre essas duas influências pode ser determinado pela expectativa do paciente, devendo ser considerada na abordagem terapêutica ${ }^{(16)}$.

Além disso, em razão da cronicidade da doença, os problemas e dificuldades relatados pelos pacientes vão de encontro à difícil tarefa de manter o tratamento ininterrupto, quando, muitas vezes, Ihes faltam condições e suporte social e familiar para fazê-lo. Isso torna imprescindível o adequado planejamento e promoção da assistência de enfermagem, para o cuidado e promoção da autonomia desses pacientes $^{(12)}$.

Somando-se a isso, estigmas e preconceitos também são apontados como fatores determinantes, para o abandono do tratamento ${ }^{(9,13)}$. Do mesmo modo, a terapêutica utilizada para o tratamento desse transtorno é responsável por afetar os níveis de concentração, insatisfação com a imagem corporal e diminuição da autoestima dos indivíduos ${ }^{(1)}$.

No que se refere à avaliação da qualidade de vida dos pacientes, o estudo demonstrou que a maioria dos 35 pacientes estudados $(77,2 \%)$ apresentou qualidade de vida regular. Outros estudos também evidenciaram que a qualidade de vida dos pacientes com transtorno bipolar é regular ou ruim ${ }^{(17)}$. Desse modo, deve-se compreender, ao se abordar a qualidade de vida dos pacientes, que essa vida é o resultado da interação de fatores complexos e multifacetados, tais como as percepções individuais de cada paciente, sua saúde, o ambiente social, cultural, financeiro, os aspectos biológicos e espirituais que os envolvem. Assim, tanto a doença pode prejudicar outras áreas que, juntas, resultam na sua qualidade de vida, como estas também podem levar à doença ou à sua estabilidade ${ }^{(4,7)}$.

Os domínios Psicológico $(68,6 \%)$ e Físico $(65,7 \%)$ foram os que mais contribuíram para a qualidade de vida regular, seguidos do domínio Meio Ambiente com pior índice para a qualidade de vida dos pacientes $(25,7 \%)$. Esse fato pode estar relacionado à fase aguda do transtorno bipolar. Em decorrência da oscilação do humor, os episódios depressivos e/ou de mania (euforia) provocam um prejuízo maior do funcionamento mental, comportamental, físico e social nos pacientes, levando-os a uma maior incapacidade geral, bem como para a execução de tarefas físicas, além do aumento de ganho de peso, fadiga e prejuízos na sexualidade, como perda de libido e impotência. Evidências mostram que a cronicidade da doença, as limitações desenvolvidas e o uso prolongado dos medicamentos estão envolvidos na pior avaliação de qualidade de vida, principalmente, nos aspectos físico e psicológico ${ }^{(18)}$.

Evidências indicam o domínio Meio Ambiente como o mais sensível a variações socioeconômicas, tendo em vista que pacientes com transtorno bipolar possuem, de modo geral, limitações e dificuldades de trabalho e, por conseguinte, acesso a atividades de lazer ${ }^{(14)}$. Esse achado aponta para a necessidade de construção de novas estratégias de reinserção social dos pacientes no mercado de trabalho e da promoção de direitos e acessibilidade à cultura e lazer.

A posologia medicamentosa utilizada no tratamento por grande parte dos pacientes que participaram do estudo foi complexa, com a associação de mais de quatro tipos de medicamentos/dia. Nesse sentido, a equipe de enfermagem deve atuar de forma ativa no sentido de promover, por meio da assistência de enfermagem, a remissão dos sintomas e a capacidade de resiliência dos pacientes, que são apontados como essenciais para a melhora da qualidade de vida, principalmente nos aspectos físico e psicológico ${ }^{(19)}$.

O domínio Relações Sociais $(37,1 \%)$ foi considerado o que mais contribuiu para uma boa qualidade de vida dos pacientes. Esse resultado mostra como a aceitação social do paciente acometido por transtorno bipolar é um fator importante para a sua adesão ao tratamento. Sendo, desse modo, de extrema importância que o preconceito social, gerado pelo estigma da doença psiquiátrica, seja fortemente trabalhado pelos profissionais da saúde mental, dentre eles os da enfermagem, quer por meio de campanhas educativas sobre a doença e seu tratamento ou pela promoção de atividades inclusivas na sociedade dos pacientes com transtorno bipolar. Desse modo, a sociedade pode se tornar mais consciente sobre o transtorno bipolar e promover, cada vez mais, a reinserção social desses pacientes de forma digna, respeitando os seus direitos.

É oportuno enfatizar que esse processo não deve se limitar apenas ao transtorno bipolar, mas também às outras doenças mentais. As relações interpessoais entre paciente, sociedade e familiares são consideradas fundamentais para a aceitação da doença e a adesão e promoção do tratamento, podendo diminuir ou prevenir o sofrimento já existente pela doença e promover a saúde mental ${ }^{(15,17)}$. 
Cabe ressaltar que, embora o estudo tenha apresentado um resultado elevado de adesão ao tratamento no momento da alta, observou-se a ocorrência do fenômeno conhecido como porta giratória, caracterizado como ciclo recidivo de internação-alta-internação(20). Apesar de não se constituir como objetivo proposto pelo estudo, observou-se, durante o período de coleta dos dados, a reinternação de 12 dos 35 pacientes avaliados. Logo, a porta giratória evidenciou a necessidade de otimizar as ações, na rede de cuidados, melhorando fluxos de atendimento, construindo parcerias e promovendo o vínculo entre as equipes.

Por fim, tona-se oportuno explicitar que as limitações deste estudo estiveram relacionadas à sua validade externa, tendo em vista que a amostra não foi probabilística. Todavia, o delineamento escolhido respondeu bem às questões norteadoras do estudo, considerando que a investigação se deu com pacientes de difícil acesso, em tratamento no serviço de saúde mental e ao se apresentarem estabilizados no momento da alta. Ademais, a referida unidade é a única do tipo III, sendo referência no atendimento à população da região Centro-Oeste de Minas Gerais.

\section{CONSIDERAÇÕES FINAIS}

A predição da adesão ao tratamento proposto, logo antes da alta do CAPS III, mostrouse elevada, sendo maior no grupo Tratamento Psicossocial e Aliança Terapêutica com os Profissionais e Serviços de Saúde Mental. O grupo Efeitos Adversos da Medicação apresentou a menor predição de adesão. A qualidade de vida mostrou-se regular, obtendo maior ganho no domínio Relações Sociais e maior prejuízo nos domínios Psicológico, Físico e Meio Ambiente. Os achados evidenciaram a necessidade de construção de estratégias que minimizem os impactos da medicação e promovam a reabilitação psicossocial do paciente, com o intuito de potencializar a adesão e melhorar a qualidade de vida. Entre as contribuições para futuros estudos, é possível destacar: a necessidade de avaliar a adesão ao tratamento, pelo paciente bipolar, com amostras envolvendo outros contextos; a sobrecarga do tratamento para os familiares e profissionais, por se tratar de uma doença crônica a qual exige dedicação e acompanhamento, torna-se importante avaliar a saúde mental dos atores envolvidos no tratamento do transtorno bipolar e a sua relação com a maior ou menor adesão do paciente ao tratamento e ao fenômeno da porta giratória, ciclo recidivo de internação-alta-internação.

\section{REFERÊNCIAS}

1 - Granek L, Danan D, Bersudsky Y, Osher Y. Living with bipolar disorder: The impact on patients, spouses, and their marital relationship. Bipolar Disord. 2016;18(2):192-9. DOI: 10.1111/bdi.12370 2 - Rowland TA, Marwaha S. Epidemiology and risk factors for bipolar disorder. Ther Adv Psychopharmacol. 2018;8(9):251-69. DOI: 10.1177/2045125318769235

3 - Scussel F, Salvadoe LC, Brandão LC, Feier G. Clinical profile of patients with bipolar disorder treated at aspecialized clinic in southern Santa Catarina. Arq Catarin Med. 2016 [citado em 28 jan 2019]; 45(4):3-10. Available in: file:///C:/Users/Fran/AppData/Local/Packages/M icrosoft.MicrosoftEdge_8wekyb3d8bbwe/TempSt ate/Downloads/133-278-1-PB\%20(1).pdf

4 - Bojic S, Becerra R. Mindfulness-based treatment for bipolar disorder: A systematic review of the literature. Eur J Psychol. 2017;13(3):573-98. DOI: 10.5964/ejop.v13i3.1138

5 - Guedes AC, Olschowsky A, Kantorski LP, Antonacci MH. Care transfer: Discharge process of users in a psychosocial attention center. Rev Eletr Enferm. 2017;19:1-9.DOI: 10.5216/ree.v19.43794 6 - Mazzaia MC, Souza MA. Adesão ao tratamento no Transtorno Afetivo Bipolar: Percepção do usuário e do profissional de saúde. Rev Port Enferm Saúde Mental 2017;17:34-42. DOI: 10.19131/rpesm. 0181

7 - Greene M, Paladini L, Lemmer T, Piedade A, Touya $\mathrm{M}$, Clark $\mathrm{O}$. Systematic literature review on patterns of pharmacological treatment and adherence among patients with bipolar disorder type I in the USA.

Neuropsychiatr Dis Treat. 2018;14:1545-59. DOI: 10.2147/NDT.S166730

8 - Jawad I, Watson S, Haddad PM, Talbot PS, McAllister-Williams RH. Medication nonadherence in bipolar disorder: a narrative review. Ther Adv Psychopharmacol 2018;8(12):349-63. DOI: $10.1177 / 2045125318804364$

9 - Averous $P$, Charbonnier E, Lagouanelle-Simeoni MC, Prosperi A, Dany L. Illness perceptions and adherence in bipolar disorder: An exploratory study. Compr Psychiatry 2018;80:109-15. DOI: 10.1016/j.comppsych.2017.10.003

10 - Chakrabarti S. Treatment-adherence in bipolar disorder: A patient-centred approach. 
World J Psychiatry 2016;6(4):399-409. DOI:10.5498/wjp.6.i4.399

11 - Marchi R. Escala clínica para prever a adesão ao tratamento: Transtorno bipolar do humor [tese]. Campinas (SP): Centro de Ciências da Vida Pontifícia Universidade Católica; 2008.

12 - Merikangas KR, Jin R, He JP, Kessler RC, Lee S, Sampson NA, et al. Prevalence and correlates of bipolar spectrum disorder in the world mental health survey initiative. Arch General Psychiatry 2011;68(3):241-51.

DOI:

10.1001/archgenpsychiatry.2011

13 - Velligan DI, Sajatovic M, Hatch A, Kramata P, Docherty JP. Why do psychiatric patients stop antipsychotic medication? A systematic review of reasons for nonadherence to medication in patients with serious mental illness. PatientPrefer Adherence 2017;11:449-68. DOI:10.2147/PPA.S124658

14 - Clemente AS, Santos WJ, Nicolato R, Firmo JOA. Stigma related to bipolar disorder in the perception of psychiatrists from Belo Horizonte, Minas Gerais State, Brazil. Cad Saúde Pública 2017;33(6):1-10. DOI: 10.1590/0102$311 \times 00050016$

15 - Stentzel U, Van den Berg N, Schulze LN, Schwaneberg T, Radicke F, Langosch JM, et al. Predictors of medication adherence among patients with severe psychiatric disorders: Findings from the baseline assessment of a randomized controlled trial (Tecla). BMC Psychiatry 2018;18(1):155. DOI: 10.1186/s12888018-1737-4

16 -Provencher MD, Bélanger M-Ė, Shriqui C, Lachance I, Bonneville S. L'éducation thérapeutique du patient pour les clientèles psychiatriques présentant un surpoids: L'exemple du programme «Wellness» développé au Québec. L'Encéphale 2016;42(3):201-7. DOI: 10.1016/j.encep.2016.01.006

17 - Murray G, Leitan ND, Thomas N, Michalak EE, Johnson SL, Jones $S$, et al. Towards recoveryoriented psychosocial interventions for bipolar disorder: Quality of life outcomes, stage-sensitive treatments, and mindfulness mechanisms. Clin Psychol Rev. 2017;52:148-63. DOI: 10.1016/j.cpr.2017.01.002

18 - Weisenbach SL, Marshall D, Weldon AL, Ryan KA, Vederman C, Kamali M, et al. The double burden of age and disease on cognition and quality of life in bipolar disorder. Int J Geriatr Psychiatry 2014;29(9):952-61. DOI: 10.1002/gps.4084

19 - Hofer A, Mizuno Y, Wartelsteiner F, Wolfgang FW, Frajo-Apor B, Kemmler G, et al. Quality of life in schizophrenia and bipolar disorder: The impact of symptomatic remission and resilience. Eur Psychiatry 2017;46:42-7. DOI: 10.1016/j.eurpsy.2017.08.005

20 - Zanardo GLP, Silveira LHC, Rocha CMF, Rocha KB. Internações e reinternações psiquiátricas em um hospital geral de Porto Alegre: Características sociodemográficas, clínicas e do uso da Rede de Atenção Psicossocial. Rev Bras Epidemiol. 2017;20(3):460-74. DOI: 10.1590/19805497201700030009

Nota: este artigo integra a dissertação de mestrado "Transtorno bipolar: fatores preditivos da adesão ao tratamento farmacológico e psicossocial e qualidade de vida" do Programa de Pós Graduação em Enfermagem da Universidade Federal de São João Del Rei (UFSJ).

Recebido em: 19/09/2019

Aprovado em: 21/04/2020

Endereço de Correspondência:

Clarice de Lourdes Enes. Rua Piauí, 780/301 - Santo Antônio CEP: 35502-031 - Divinópolis (MG).

e-mail: clarice.enes@gmail.com 